\title{
Keeper, Fouls, Assists - Anglizismen in der Sprache des Fußballs, Eishockeys und Handballs
}

\author{
Marco WINKLER
}

Gewidmet dem Sieger der EHF European League und des IHF Super Globe 2021, dem SC Magdeburg

\begin{abstract}
Keeper, fouls, assists - Anglicisms in the language of football, ice hockey and handball

Nowadays, anglicisms seem to be indispensable in the language of sport - as in other professional languages. However, the sports considered in this study differ greatly as to their origin and the importance of teams from English-speaking regions (England regarding football, North America regarding ice hockey, whereas in handball there is no English-speaking influence). This results in linguistic differences regarding the frequency and professional relevance of anglicisms in each sport. These differences are investigated and compared taking into account morphological, semantic and sociolinguistic aspects.
\end{abstract}

Keywords: Anglicisms, sports language, professional language

DOI: $10.15452 /$ StudiaGermanistica.2021.29.0004

\section{Start}

Anglizismen begegnen uns häufig in der Fach- und Alltagssprache. Einige Menschen nutzen sie exzessiv, andere hingegen stehen ihnen kritisch gegenüber. Auf jeden Fall ist ihre Verwendung vielfach sinnvoll und nicht zu umgehen. Auch die Sprache des Sports prägen sie - schon das Wort Sport selbst ist ein Anglizismus, der erst in den 20er Jahren des 19. Jahrhunderts ins Deutsche kam. Damit haben auch vehemente Kritiker eines übermäßigen Anglizismengebrauchs, wie Wolf Schneider, kein Problem. Er lobt gerade die kurzen und kraftvollen Wörter der englischen Sprache - ,und da, bei ihren einsilbigen Wörtern, haben wir uns zu Recht am kräftigsten bedient" (Schneider 2008:16), wozu für ihn neben Sport auch fair, fit, Fan, Spurt, Start, Team sowie einige Zweisilber wie clever oder Training gehören, also Wörter, die uns, nicht nur in der Sportsprache, immer wieder begegnen.

Für wichtige Hinweise zur Sportsprache danke ich Armin Burkhardt. Für lange Diskussionen über Sport, Sportsprache und deutsch-tschechische Unterschiede bin ich Jana Novotná und Radovan Lakosil dankbar. 
Wie sieht es aber in der Fachterminologie einzelner Sportarten aus? Dieser Frage soll in der vorliegenden Studie mit Blick auf die drei wichtigsten Mannschaftssportarten in Deutschland nachgegangen werden. Den Anfang macht dabei der Fußball, er ist heute die unumstritten populärste Sportart im Land, und seine Herkunft aus England hatte und hat Einfluss auf seine Fachsprache. Er ist auch die älteste dieser drei Sportarten in Deutschland, kam Mitte der 70er Jahre des 19. Jahrhunderts ins Land und breitete sich rasch aus. Allerdings war er in den ersten Jahrzehnten, nicht zuletzt auf Grund seiner englischen Herkunft, nicht unumstritten (vgl. Burkhardt, 2006, 2008b, 2015). Mit Eishockey kommt eine Sportart hinzu, deren Terminologie stark durch die Herkunft aus einer anderen englischsprachigen Region, aus Nordamerika, bestimmt wird. Es wird in Deutschland seit den 90er Jahren des 19. Jahrhunderts gespielt (vgl. Müller 2000; Bröker 2013, 2016). Im Handball hingegen war nie ein englischsprachiges Land prägend. Er ist der jüngste Sport in der Auswahl, wurde Ende des 19. Jahrhunderts in Dänemark entwickelt und kam während des 1. Weltkriegs nach Deutschland, wo er ursprünglich als Mannschaftssport für Frauen angesehen wurde (vgl. Wesemeyer 2009:21 ff.). Eine Gegenüberstellung dieser drei Sportarten hinsichtlich des Vorkommens von Anglizismen in der Fachsprache scheint also - vor dem Hintergrund so unterschiedlicher Entwicklungen - lohnend. Dazu wird ein Überblick über das Repertoire englischer Ausdrücke, zu ihrem Status, ihrer Verwendung und Herkunft gegeben. Auf dieser Grundlage erfolgt eine Analyse und ein Vergleich des Vorkommens von Anglizismen in den drei Sportarten. Eine vollständige Auflistung und ausführliche Beschreibung ihrer Bedeutung ist dabei nicht angestrebt.

In der vorliegenden Untersuchung geht es um den Fachwortschatz in drei Bereichen der Sportsprache: Sportfachsprache, Sportjargon und Mediensprache des Sports (vgl. Burkhardt 2006:55 f.). Zur Sportfachsprache gehören beispielsweise die Terminologien in Regelwerken oder in Lehrbüchern und Handbüchern für Trainer. Der Sportjargon umfasst die Äußerungen von Trainern, Spielern und anderen Sachkundigen über den Sport, also die Sprache im Training und während des Spiels, Kommentare zu Spielen in Interviews und Pressekonferenzen usw. Zur Mediensprache des Sports gehört die Berichterstattung in Zeitungen oder im Fernsehen, also Spielberichte, Kommentare zu Liveübertragungen $u$. ä.

Im Zentrum der vorliegenden Untersuchung steht die deutsche Sportsprache in Deutschland. Unterschiede zum österreichischen oder schweizerischen Deutsch werden nicht ausführlich diskutiert, allerdings wird teilweise auf sie hingewiesen. Bei einigen Fachausdrücken fließen kontrastive Vergleiche zum Tschechischen ein. Mit dieser konfrontativen Sicht wird eine veränderte Perspektive auf das Deutsche möglich, die lexikalischen Besonderheiten klarer hervorhebt. Auf die Bedeutung solcher kontrastiver Betrachtungen weist Burkhardt $(2008,2021)$ hin.

Im Text werden für die Anglizismen Belege aus authentischen Kontexten angeführt, die den tatsächlichen Sprachgebrauch wiedergeben. Hierfür wurde ein Korpus zusammengestellt, aus dem eine Belegsammlung erstellt wurde. Für die Fachsprache des Sports bilden die Regelbücher der drei Sportarten ( $\mathrm{ReBuFu}, \mathrm{ReBuEi}, \mathrm{ReBuHa})$ die Grundlage, hinzu kommen die Broschüre handballtraining 02/2009 (HaTr) und das Trainerhandbuch Fußball. Kondition - Technik - Taktik und Coaching von Bisanz und Gerisch (FuKTTC). Als sportspezifische Medien wurden die Zeitschrift Handballwoche (HaWo), das Portal handball-world (HaWd), das Portal hockeyweb (HoWe) und die Zeitschrift Kicker in der Printausgabe (KiPr) und der Online-Version (KiOn) genutzt. Weiterhin wurden die Online-Ausgaben des Spiegel (SP), der Frankfurter Allgemeinen Zeitung (FAZ) und der Süddeutschen Zeitung (SZ) verwendet. Für historische Fragestellungen wurden der Aufsatz Deutsche Kunstausdrücke des Fußballspieles (KuAu) von Konrad Koch aus dem Jahre 1903 und der Führer durch die Sportsprache von 1935 (FDS) herangezogen.

\section{Dribbling in der Box - Fußball}

Als der Fußball in den 70er Jahren des 19. Jahrhunderts nach Deutschland kam, hatte er es nicht leicht. Er wurde als englische Krankheit oder Fußlümmelei beschimpft, die nicht mit deutschen Tugenden vereinbar sei (z. B. Planck 1898). Als deutscher Sport überhaupt galt Turnen, das schon 
seit den Befreiungskriegen gegen die französische Besatzung hohes nationales Ansehen besaß. Nicht zuletzt wegen des national gesinnten Umfeldes nach der Reichsgründung von 1871 und der Tatsache, dass England immer weniger als Verbündeter, sondern zunehmend als internationaler Konkurrent wahrgenommen wurde, trieben Pioniere dieser Sportart, insbesondere Konrad Koch, die Verdeutschung seiner Fachterminologie voran; diese Entwicklung wird ausführlich von Burkhardt (2006, 2008b, 2015) dargestellt. So wurden englische Ausdrücke wie Penalty, Offside, Corner oder Goal konsequent durch deutsche Wörter ersetzt. Auf Grund der strukturellen Ähnlichkeit des Deutschen und Englischen waren auch einige Lehnübersetzungen naheliegend (Halbzeit für half time oder Abseits für Offside). In anderen Sprachen, selbst im Deutschen in Österreich und der Schweiz, wurden in stärkerem Maße die englischsprachigen Bezeichnungen übernommen (vgl. Glauninger/ Graf 2009; Ammon 1995). Auch im Tschechischen sind gól, ofsajd und penalta die gebräuchlichen Fachtermini. Sogar im Französischen, sonst nicht unbedingt für eine begeisterte Übernahme von Anglizismen bekannt, sind viele englische Ausdrücke in der Fußballsprache zu finden, z. B. football, corner, match null, penalty, shoot (vgl. Eisenberg 2011:138).

Der wahrscheinlich einflussreichste Text aus der Anfangszeit des deutschen Fußballs, in dem Vorschläge für eine Verdeutschung der Terminologie unterbreitet wurden, ist Deutsche Kunstausdrücke des Fußballspieles von Konrad Koch aus dem Jahre 1903 (KuAu). Viele Ausdrücke, die dort in einem Glossar zu finden sind, haben sich auch durchgesetzt und sind heute fester Bestandteil der Fußballsprache in Deutschland:

$\begin{array}{lll}\text { forwards } & = & \text { Stürmer } \\ \text { centre-forward } & = & \text { Mittelstürmer } \\ \text { corner } & = & \text { Ecke } \\ \text { corner-kick } & = & \text { Eckball, Eckstoß } \\ \text { drawn } & = & \text { unentschieden } \\ \text { goal } & = & \text { Tor, Mal } \\ \text { half time } & = & \text { Halbzeit } \\ \text { kick-off } & = & \text { Anstoß } \\ \text { off side } & = & \text { abseits } \\ \text { penalty-goal } & = & \text { Straftor } \\ \text { penalty-kick } & = & \text { Strafstoß } \\ \text { shoot } & = & \text { Schuß } \text { Stoß) aufs Tor } \\ \text { to shoot } & = & \text { schießen } \\ \text { tripping } & = & \text { Beinstellen }\end{array}$

Andere Vorschläge wiederum konnten sich nicht durchsetzen und sind heute teilweise unbekannt, oder sie existieren neben der englischen Bezeichnung:

$\begin{array}{lll}\begin{array}{ll}\text { captain } \\ \text { combination }\end{array} & = & \text { Spielwart (Spielkaiser) } \\ \text { to centre } & = & \text { nach der Mitte stoßen } \\ \text { to dribble } & = & \text { treiben } \\ \text { fair } & = & \text { anständig, ehrlich } \\ \text { foul } & = & \text { ungehörig, unehrlich } \\ \text { goal-keeper } & = & \text { Torwächter } \\ \text { match } & = & \text { Wettspiel } \\ \text { to pass } & = & \text { abgeben, zuspielen } \\ \text { referee } & = & \text { Schiedsrichter } \\ \text { to tackle } & = & \text { fassen, halten } \\ \text { team } & = & \text { Mannschaft, Riege } \\ \text { unfair } & = & \text { ungehörig, unfein }\end{array}$


Zum Teil haben sich auch völlig neue Benennungen ergeben, wie z. B. Flanke für to centre. Im Tschechischen hingegen hat sich der Anglizismus erhalten als centr ,zur Mitte' oder centr ze strany ,zur Mitte von der Seite'.

Bei einigen Wörtern der Sportsprache wird im Deutschen nicht mehr wahrgenommen, dass es sich um Anglizismen handelt. So wurde das Wort Pass bzw. das entsprechende Verb passen aus dem Englischen pass ,Übergang, Durchgang` entlehnt. Vorschläge für eine Verdeutschung in der Fußballsprache gab es zwar mit abgeben oder zuspielen, und diese kommen auch vereinzelt vor, konnten sich aber - im Unterschied zu Tor, Schuss oder Eckball - nicht durchsetzen. Inzwischen ist das Wort voll in den deutschen Sportwortschatz integriert und dient als Grundwort in vielen Komposita (Rückpass, Querpass, Traumpass usw.), die im Fußball, aber auch im Eishockey oder Handball, verwendet werden:

An allen vier Eintracht-Treffern war der 27-jährige Kapitän beteiligt, bereitete das erste Tor per Traumpass vor, traf zweimal selbst und leitete ein weiteres ein. (KiPr 12.04.2021, 62)

Dann setzte er mit einem Steilpass Sané in Szene, der von der linken Seite auf Havertz passte. (KiPr 29.03.2021, 23)

Auf solche Entlehnungen in der Sportsprache, die „als Anglizismen nicht mehr ersichtlich“ sind, weist Born (2009:19) hin. Die von ihm erwähnte ,einfache Möglichkeit, durch Anhängen des Suffixes -en aus dem Englischen Verben zu übernehmen“" (Born 2009:19), die in der Sportsprache häufig zu finden ist (z. B. kicken, dribbeln, sprinten), ist auch beim Verb passen zu sehen. Ähnlich sieht es mit dem Anglizismus Foul für unsportliches, unfaires oder gefährliches Verhalten im Spiel (ReBuFu Regel 12) und das entsprechende Verb foulen aus. Anglizismen sind also in der Sportsprache hinsichtlich ihrer Integration in den Wortschatz zu unterscheiden (vgl. Eisenberg 2011:21 f.). Es gibt Wörter, die bereits in die deutsche Sprache integriert wurden, also zum Kernwortschatz gehören oder zumindest in diesen übergehen (Pass, Foul, Konter, Check). Diesen merkt man ihre Herkunft aus der englischen Sprache oftmals gar nicht mehr an, vor allem in Fällen, ,in denen die entlehnten Wörter schon bei der Übernahme so aussahen wie Wörter des Kernwortschatzes“ (Eisenberg 2011:21). Es fällt auf, dass es sich dabei häufig um Einsilber handelt. Auf den Umstand, dass diese als besonders integriert gelten und auch bei Kritikern von Anglizismen eher akzeptiert sind, weist Eichinger (2008:75 f.) hin (vgl. auch die bereits angeführte Aussage von Wolf Schneider).

Auch Deutschlernen mit Kick (Sporkmann/Zygowski 2005), ein DaF-Lehrwerk für die Niveaustufen A1-B1, das Fußball thematisiert, kommt nicht ohne dribbeln, foulen und kicken aus. Und in modernen Trainingslehrbüchern ist das Dribbeln ebenfalls unverzichtbar:

Das Dribbeln ist die Grundtechnik, die von allen Spielern, angefangen bei den Kleinsten bis hin zu den Profis, gerne angewendet wird. (FUKTTC 494)

Das Dribbling bietet dem Spieler am Ball vielfältige Möglichkeiten, Finten einzusetzen, wobei die Täuschung das wesentliche Kriterium darstellt. (FUKTTC 498)

Schon 1935 war es etabliert, wie ein Blick in den Führer durch die deutsche Sportsprache zeigt:

Dribbeln ist die Kunst, einen Gegner, wenn es nicht anders ist, mit teuflischen Täuschungsmanövern zu umgehen. (FDS:8)

Auch das Tackling, das Koch noch von deutschen Fußballplätzen verbannen wollte, ist heute in der Fußballsprache präsent:

Mit Tackling werden verschiedene Techniken der Ballabnahme bezeichnet. Die konzentrierte Beobachtung von Ball und Gegner, das präzise Abpassen des richtigen Zeitpunkts zum Eingreifen und ein schneller Bewegungsablauf sind wichtige Voraussetzungen für ein erfolgreiches Tackling. (FUKTTC 564) 
Bezüglich eines speziellen „Sprint-Tackling”-Trainings ist den Spielern bewusst zu machen, dass einem Tackling häufig ein Stellungsfehler vorausgeht. Deshalb sollte das Tackling immer das Mittel letzter Wahl sein. (FUKTTC 307)

Neue Anglizismen kommen immer wieder in die Fach- und auch Umgangssprache mit der Herausbildung von fachlichen Neuerungen. So brachten Entwicklungen in der Taktik des Fußballs neue Fachausdrücke mit sich. Bei der Weltmeisterschaft 1974 sorgte die niederländische Nationalmannschaft mit einer neuen Spielweise für Aufsehen - dem Pressing. Dieses Konzept revolutionierte das Spiel und gehört mittlerweile zum Standardrepertoire des modernen Fußballs (vgl. Biermann 2019):

Wenn zwei Abwehrspieler aggressiv gegen den Ballbesitzer spielen und die Spieler in seiner näheren Umgebung eng gedeckt werden, dann wird das heute mit dem Begriff Pressing bezeichnet. (FUKTTC 658)

Das Mittelfeldpressing ist eine Möglichkeit, schon früh mit aggressiven Abwehraktionen zu beginnen und den Gegner vom eigenen Tor fernzuhalten. (FUKTTC 663)

Typisch für diese Spielweise ist, dass derjenige Gegenspieler, der in Ballbesitz ist, attackiert wird. So wird die gegnerische Mannschaft unter Druck gesetzt und am Spielaufbau gehindert. Später kam das Forechecking hinzu, das ursprünglich aus dem Eishockey stammt. Dabei wird der Gegner bereits tief in der eigenen Hälfte gestört, um Angriffsversuche frühzeitig zu unterbinden oder zumindest zu erschweren.

Hertha tat sich schwer, dem aggressiven Forechecking des Tabellenführers zu entkommen. (KiOn 27.10.2018)

[...] weiß Schmidt, der aber auch eine Schwachstelle ausgemacht hat: ,, Sie verteidigen oft hoch mit Angriffspressing und Forechecking, auch manchmal Mann gegen Mann. Wenn man diese Situationen richtig lösen kann, gibt es Raum für uns. "(KiOn 18.03.2021)

Hierzu gibt es keine deutsche Entsprechung, Angriffsverteidigung o. ä. hat sich nicht durchgesetzt. Im Tschechischen dagegen gibt es neben dem Anglizismus forčekink auch napadání ,Angriff, Überfall'. Ab den 2000er Jahren sorgte das Gegenpressing für Aufsehen, bei dem sofort nach Ballverlust auf Pressing umgeschaltet wird, um den Ball zurückzugewinnen.

Hochleistungs-Pressing und-Gegenpressing ist spätestens seit seiner BVB-Zeit typisch für Klopp, allerdings musste er ob immer tiefer stehender Gegner immer mehr Lösungen mit längeren Ballbesitzphasen erarbeiten. (KiPr 04.03.2021, 32)

„Nein“, sagt Hartenbach, ,, wir überlassen den Ball nicht absichtlich dem Gegner, um ihn im Pressing und Gegenpressing erobern zu können. " (KiPr 25.02.2021, 25)

Den Spielaufbau macht Pressing zwar schwierig, aber es gibt auch Gegenmittel, wie z. B. OneTouch-Fußball. Dabei behält ein Spieler den Ball nicht, sondern passt ihn direkt zu einem Mitspieler usw. Es gibt also nur einen Kontakt, der gleich die Abgabe des Balls bedeutet:

Mit spektakulärem One-Touch-Fußball kombinierte speziell das Quartett Kostic, Jovic, Kamada und Silva binnen weniger Minuten einen 4:1-Vorsprung heraus. (KiPr 22.03.2021, 40)

Ein weiteres Mittel gegen das Pressing und überhaupt gegen eine stabile Abwehr der Gegner sind Chip-Bälle, also hohe Pässe über die Abwehr:

Der Unterschied bis dahin in einem offenen Spiel, in dem Hertha zu guten Umschaltmomenten kam und die Gäste die frühe Berliner Pressinglinie mit langen Chipbällen zu überspielen versuchten. (KiPr 22.02.2021, 30)

Mit einem Chip-Ball kann auch der Torhüter überspielt werden, und die Kurzform Chip sowie das abgeleitete Verb chippen sind ebenfalls zu finden: 
Atik schickte Conteh, der den Ball mit einem feinen Chipball ins Tor versenkte (2.). (KiOn 28.08.2021)

Herthas Matheus Cunha hatte dabei kurz vor Schluss die große Möglichkeit zum Ausgleich, verfehlte das Tor bei seinem Chip über den Münchner Keeper lediglich um ein paar Zentimeter. (KiPr 08.02.2021, 32)

Beim 1:0 startete der Angreifer genau im richtigen Moment, um dann den Ball im Stile von Raul gekonnt über den Keeper zu chippen. (KiPr 11.01.2021, 25)

Spezielle Arten von Schüssen sind der Dropkick und der Volleyschuss:

Dabei ergeben sich die Variationen, den Ball auf dem Boden ruhend oder rollend zu treffen - den Flugball volley, den aufspringenden Ball unmittelbar nach der Bodenberührung als Dropkick. (FuKTTC 522)

Hoch einfallende oder springende Bälle können gut dosiert volley oder als Dropkick weitergeleitet werden. (FuKTTC 516)

Der Dropkick ist insbesondere wegen seiner Härte sehr gefährlich:

Der Dropkick findet Anwendung als Zuspiel, als Torschuss aus verschiedenen Entfernungen und im Bereich der Abwehr als Abschlag des Torhüters sowie als Befreiungsschlag durch Abwehrspieler. Beim Dropkick mit dem Vollspann wird der Ball zentral getroffen und erhält dadurch die besondere Schärfe. Der Dropkick mit dem Innenspann wird selten angewendet. Besonders effektvoll ist der Dropkick mit dem Außenspann. (FuKTTC 525)

Volleyschüsse treten ebenfalls in verschiedenen Situationen auf, in der Abwehr, als Pass oder als Torschuss:

Volley und mit vollem Risiko knallte Neidhart den Ball nach super Hacken-Vorarbeit von Nik Omladic mit seinem rechten Fuß in den linken Torwinkel. (KiPr 22.02.2021, 80)

Johannes van den Bergh, unermüdlich auf der linken Bahn unterwegs, leitete mit seiner Volleyflanke den Siegtreffer ein. (KiPr 14.05.2021, 39)

Als Kick and Rush wird eine Spielweise bezeichnet, bei der eine Mannschaft nicht durch systematisches Aufbauspiel über das Mittelfeld einen Angriff aufbaut, sondern aus dem eigenen Verteidigungsbereich den Ball weit in die gegnerische Hälfte spielt. Dort sollen dann die Stürmer den Ball unter Kontrolle bringen und gefährliche Angriffsaktionen herausspielen. Diese Spielweise ist nicht unumstritten und wird von vielen Trainern und Fans abgelehnt:

Daneben glänzte der physisch starke Stürmer auch als ballsichere Anspielstation, die im Kick-andrush des SVD Bälle gekonnt festmachte und Lücken für die eigenen Mitspieler riss. (KiOn 24.07.2016)

Die Zeiten von Kick and Rush sind weitgehend vorbei. (KiPr 21.09.2017, 37)

Dieser sehr modern klingende Anglizismus könnte vermuten lassen, dass es sich um eine neue Taktik handelt; dem ist aber nicht so, wie ein Blick in die Fußballsprache von 1935 verrät:

Mit unverhohlener Mißachtung wird in der Runde der Fußballweisen festgestellt, dass diese oder jene Elfnoch das vorsintflutliche ,kick and rush " betreibe, was mit ,Wald- und Wiesenfußball " allzu naiv übersetzt ist, denn auch , kick and rush" ist ein Stil, wenn man auch ganz einfach nur den Ball dort wieder sucht, wo man ihn hingetreten hat. (FDF 5 f.)

Im modernen Fußball gewann diese Spielweise wieder etwas an Bedeutung mit dem Gegenpressing, denn ein geordneter Spielaufbau über das Mittelfeld wurde so immer schwieriger, und mit Pässen weit in die gegnerische Hälfte konnte dieses schnell überwunden werden. Kick and rush scheint ein typischer Anglizismus zu sein. Dass diese Bezeichnung vor allem auf dem europäischen Kontinent zu finden ist, während in England die Bezeichnung Long Ball vorherrscht, wird dabei gern übersehen. 
Der Strafraum, der sich 16 Meter vor dem Tor erstreckt, wird in der modernen Fußballsprache wegen der viereckigen Form als Box bezeichnet:

$\mathrm{Zu}$ oft enden die Angriffsvorträge schon am Strafraum des Gegners, es herrscht Flaute in der Box. (KiPr 22.02.2021, 50)

Der Strafraum ist sein Zuhause: Albert Bunjaku fühlt sich in der Box sehr wohl. (KiPr 25.03.2021, 42)

„Wir sind dort seit Wochen nicht gut, weil wir uns nicht gut genug in der Box bewegen “, haderte der 55-Jährige vor zwei Wochen. KiPr 06.04.2021, 39)

Ein Mittelfeldspieler, der in dem großen Bereich vom eigenen bis zum gegnerischen Strafraum, also zwischen beiden Boxen, agiert, wird als Box-to-Box-Spieler bezeichnet. Befindet er sich näher an der eigenen Box, hat er defensive Aufgaben, näher an der gegnerischen Box ist er wiederum offensiv aktiv. Er muss also Verteidigung und Angriff gleichermaßen beherrschen:

Denn nach Babbels Urteil ist Goretzka ,der weltbeste Box-to-Box-Spieler. Da sehe ich keinen besseren". (KiPr 12.04.2021, 13)

Matarazzo bezeichnet ihn als „Box-to-Box-Spieler, ein guter Pressingspieler, der in der Lage ist, zwischen den Linien die Bälle abzuholen und torgefährlich zu sein “. (KiPr 29.03.2021, 45)

Besondere Anglizismen sind Tank und auch das abgeleitete Verb (durch)tanken. Es handelt sich hierbei um Metaphern mit einem klar militärischen Bezug. Damit werden Angriffsspieler beschrieben, die physisch sehr stark sind und sich gut durch die gegnerische Abwehr bewegen können. Da diese Ausdrücke im Fußball, Eishockey und Handball gleichermaßen vorkommen, sollen gleich hier Belege aus allen drei Sportarten angeführt werden:

Entscheidend in diesem Heimspiel waren das zwischenzeitliche wie etwas überraschende 1:0, ehe kurz nach dem kassierten 1:1 die feurige Antwort von Sturmtank Hennings gab. (KiOn 24.01.2018)

Hendrik Weydandt startete in der eigenen Hälfte durch und tankte sich bis in den Strafraum durch. (KiOn 12.02.2021)

Der finnische Sturmtank absolvierte 210 Pflichtspiele für den ESVK, erzielte dabei 66 Tore und 96 Torvorlagen. (HoWe 08.04.2020)

Louis Latta tankte sich von außen in den Slot durch und schloss trocken ins obere Eck zum 1:0 ab. (HoWe 11.12.2020)

Der Bundestrainer hat ihn ins Herz geschlossen. Und der Sturmtank dankte es beim Supercup mit imponierenden Vorstellungen. (HaWd 04.11.2013)

Weber tankte sich durch und traf zum 25:22 (44.), im Gegenzug gelang Bitter die sechste Parade. (HaWd 21.01.2021)

Sehr glücklich sind Spieler, die in einem Spiel drei Tore nacheinander erzielen, also einen Hattrick schaffen. Dabei gehen die Meinungen darüber auseinander, ob dies nur in einem Spiel oder in einer Halbzeit geschafft werden muss. Der englische Ausdruck hat-trick entstand ursprünglich im späten 19. Jahrhundert im Cricket. Einem Spieler, der drei Würfe in Folge zu erfolgreichen Wickets verwandelte, wurde für dieses Kunststück (trick) als Auszeichnung ein Hut (hat) überreicht (Stevenson 2010). Da diese Bezeichnung nicht nur im Fußball verwendet wird, sondern auch im Eishockey, folgen hier Belege für beide Sportarten:

Eden Hazard erzielte drei Treffer - sein zweiter Hattrick für den Klub von der Stamford Bridge. (KiPr 17.09.2018, 56)

Im Oktober 2013 hatte der Ex-Berliner mit drei Treffern in acht Minuten in Nürnberg Gerd Müller als schnellsten Hattrick-Schützen der Bundesliga abgelöst. (KiPr 17.09.2018, 71) 
Der deutsche Superstar Leon Draisaitl drückte dem Spiel mit einem Hattrick seinen Stempel auf. (HoWe 08.04.2021)

Pastrnak ist damit der zweite aktive Spieler, der neun Hattricks (acht in der Hauptrunde, einen in den Play Offs) vor seinem 24. Geburtstag erzielt hat. (HoWe 13.02.2020)

Weiterhin wird von einem Titelhattrick gesprochen - damit ist der Gewinn eines Titels (Meisterschaft, Pokal) in drei aufeinanderfolgenden Spielzeiten gemeint. Dieser Ausdruck ist im Fußball, Eishockey und auch im Handball üblich:

Bayer 04 Leverkusen steht mit drei Titeln in der DHB-Bestenliste. Mit einem Meister-Hattrick in den Jahren 2013 bis 2015. (HaWo 23.05.2017, 39)

Damit gewann der Champions-League-Sieger zum dritten Mal hintereinander das Endspiel beim Final Four gegen die Flensburger. Es ist bereits der dritte Hattrick, 1998-2000 und 2007-2009 war dem THW bereits das gleiche Kunststück gelungen. (HaWd 14.04.2013)

Für die Fußballsprache ist eine Entwicklung hinsichtlich des Anglizismengebrauchs zu verzeichnen. Die Bemühungen um eine deutschsprachige Terminologie in der Anfangsphase waren teilweise erfolgreich. Diejenigen englischen Ausdrücke, die erhalten blieben, fanden bald Eingang in den Kernwortschatz des Deutschen. Sie sind inzwischen eher als Lehnwörter anzusehen oder entwickeln sich zumindest zu solchen (fair, Foul, Match, Team). In den letzten Jahrzehnten sind wiederum neue englische Ausdrücke hinzugekommen. Insgesamt finden sich jedoch relativ wenig Anglizismen - nach Burkhardt (2008a:228), „machen sie nicht wesentlich mehr als ein Prozent des Fußballwortschatzes aus“, das „ergibt sich aus der überwiegend empirisch ermittelten Lemmaliste“ seines Wörterbuchs der Fußballsprache (Burkhardt 2006a). Allerdings ist ihre Relevanz nicht zu unterschätzen, da sie in großem Maße die moderne Taktik des Spiels und Techniken betreffen (Pressing, Forechecking, One-Touch-Fußball, Box-to-Box-Spieler, Chip-Ball, Dropkick) und zur Fachsprache im engeren Sinne gehören.

\section{Penaltys gegen Cracks wegen Checks - Eishockey}

Der große nordamerikanische Einfluss auf das deutsche Eishockey und seine Sprache ist nicht zu übersehen. In Berichten über Spiele der deutschen Nationalmannschaft werden beispielsweise Spieler, die in der nordamerikanischen National Hockey League (NHL) aktiv sind, besonders hervorgehoben und als NHL-Profi (-Crack, -Star, -Torjäger, -Stürmer, -Verteidiger, -Goalie, -Torhüter, -Torwart, -Keeper, -Jungstar) bezeichnet, was als Gütesiegel zu verstehen ist. Das kommt dem journalistischen Hang zur Verwendung von Synonymen entgegen, kann in Auswüchsen aber auch zu Absurditäten führen. So verbreitete die ,Deutsche Presse-Agentur' am 09.12.2017 einen Bericht über den Spieltag der NHL mit der Überschrift Washington gewinnt ohne NHL-Keeper Grubauer, der von ,BILD‘ und anderen deutschen Zeitungen übernommen wurde. Ist es eine Überraschung, dass in den Klubs der NHL NHL-Spieler tätig sind? Vermutlich stand in dem Spiel ein anderer NHLKeeper im Tor. Selbst für die Beschreibung der deutschen Nationalmannschaft sind Anglizismen gebräuchlich:

Am Montag landeten die DEB-Kufencracks einen richtigen Coup und schlugen Kanada nach beeindruckender Leistung mit 3:1 (KiOn 24.05.2021)

Auch bei den Bezeichnungen der Funktionen im Team finden sich Anglizismen. Der Verantwortliche für die Mannschaftsaufstellung, die Spieltaktik, die Kommunikation mit den Schiedsrichtern usw., also der Cheftrainer, wird in der deutschen Fassung des Regelbuches offiziell als Coach bezeichnet. Der Coach hat die Aufgabe, Teams oder Einzelpersonen voranzubringen und zu lenken. In Bezug auf Sport wird das Wort im Englischen erst seit der zweiten Hälfte des 19. Jahrhunderts verwendet; es wurde übernommen aus dem Bildungswesen, wo private Tutorinnen und Tutoren so genannt wurden. Ursprünglich hatte das Wort jedoch eine andere Bedeutung. Es wurde im 16. Jahrhundert vom 
französischen coche ,Kutsche“ entlehnt, das wiederum vom gleichbedeutenden ungarischen Wort kocsi stammt (wie auch deutsch Kutsche). Als Bezeichnung für Kutschen, Busse oder Eisenbahnwaggons ist es im Englischen noch heute gebräuchlich (Stevenson 2010). Auch Team kommt aus diesem Bereich; im Altenglischen wurde ein Gespann von Zugtieren als tēam bezeichnet (Stevenson 2010).

In der Mediensprache sind Bezeichnungen der Spieler als Cracks, von Torjägern als Goalgetter oder Topscorer oder der Schiedsrichter als Referee oder Ref häufig zu finden. Vor allem fällt die Benennung des Torhüters als Goalie auf, denn Goal für ein erzieltes Tor ist in der Sportsprache in Deutschland nicht üblich. Die Untersuchung eines Korpus mit 61 Zeitungsartikeln (Winkler 2018:117) ergab, dass Goal nur 2-mal in der Verbindung Garbage goal und einmal in Goalgetter zu finden ist. Für Goalie und andere Bezeichnungen für Torhüter ergab sich folgendes Bild:

\begin{tabular}{|l|l|l|l|l|}
\hline Goalie & Keeper & Torwart & Torhüter & Schlussmann \\
\hline 17 & 30 & 52 & 52 & 12 \\
\hline
\end{tabular}

Die beiden Anglizismen kamen zusammen 47-mal vor, die deutschen Bezeichnungen überwogen jedoch mit 116. Zum Vergleich mit Fußball und Handball wurden die Ausgaben 27-35/2021 des Kicker (alle im April 2021 erschienenen Hefte) und die Ausgaben 1-9/2018 der Handballwoche (alle im Januar und Februar 2018 erschienenen Hefte) ausgewertet. In allen Untersuchungen wurde auch das Vorkommen in Zusammensetzungen (z. B. Nationaltorhüter, Top-Keeper) berücksichtigt.

\begin{tabular}{|l|l|l|l|l|l|}
\hline & Goalie & Keeper & Torwart & Torhüter & Schlussmann \\
\hline Kicker & 0 & 111 & 90 & 61 & 9 \\
\hline Handballwoche & 1 & 33 & 67 & 299 & 9 \\
\hline
\end{tabular}

Im Kicker kommen die Anglizismen mit einem Anteil von 40,96 \% deutlich häufiger als in den Texten zum Eishockey (28,83 \%) vor. Im Handball gibt es mit 8,31 \% die wenigsten. Dass Keeper in allen drei Sportarten regelmäßig zu finden ist, liegt auch an der Bildungsweise des Wortes und den Parallelen der Wortbildung im Englischen und Deutschen. Personenbezeichnungen, die mit er als Nomen Agentis aus Verben ,mit der Bedeutung, Person, die die vom Verb bezeichnete Tätigkeit ausübt ${ }^{\text {“" }}$ abgeleitet werden, bilden ,,bei den Anglizismen wie im Kernwortschatz die größte Gruppe von Derivaten“ (Eisenberg 2011:262). Diese Anglizismen sind im deutschen Wortschatz „voll integriert“ (Eisenberg 2011:230) als Maskulina mit starker Flexion. Dass dem er ,eine erhebliche integrative Kraft“" zukommt, zeigt sich nach Eisenberg (2011:218) auch ,an weiteren Ableitungen, die auf seiner Basis möglich sind“, wie beispielsweise den entsprechenden weiblichen Formen auf in:

Plötzlich lief THC-Keeperin Jana Krause zur Bestform auf. (HaWo 14.02.2017, 31)

Mit Clara Woltering, Katja Kramarczyk und Dinah Eckerle hat das deutsche Team drei herausragende Keeperinnen (HaWo 28.11.2017, 15)

Für Goalie trifft das nicht zu. Das Wort entspricht keinem deutschen Wortbildungsmuster und erscheint daher in Bezug auf den Kernwortschatz fremd.

Die wichtigste Aufgabe der Torhüter ist das Verhindern von Toren - solche gehaltenen Torschüsse werden als Saves bezeichnet:

Highlight des zweiten Drittels war ein spektakulärer Save von Youngster Tim Stützle, der mit ganzem Körpereinsatz seine Mannschaft vor einem Rückstand rettete. (HoWe 13. November 2019)

Mathias Niederberger konnte mit 31 Saves alle Schüsse des Gegners erfolgreich abwehren und blieb bis zum Schluss ohne Gegentreffer. (HoWe 19.10.2019)

Die Bezeichnung Save ist im Deutschen auf Eishockey beschränkt. Im Fußball oder Handball kommt sie nur vereinzelt vor:

„Der Save von Tego war wichtig“, lobte Amos Pieper, weiteres Mitglied des Bollwerks, seinen Rückhalt. (KiPr 19.04.2021, 39) 
M. E. handelt es sich jedoch, gerade wegen der Kürze, um einen guten Kandidaten für einen Anglizismus, der zukünftig auch in die Sprache dieser Sportarten eingehen könnte.

Verhindern die Torhüter während des gesamten Spiels erfolgreich Gegentore, freuen sie sich über einen Shutout:

So konnte sich Kinkaid über einen Shutout freuen. (HoWe 07.05.2018)

Die USA ließen keinen Gegentreffer zu, sodass sich Keith Kinkaid über einen Shutout freuen konnte. (HoWe 06.05.2018)

Der heute am weitesten verbreitete Stil der Goalies ist der Butterfly-Stil:

In Nordamerika war in den 90er Jahren für Torhüter eine neue Ära angebrochen: Der Kanadier Patrick Roy ließ sich, anstatt aufrecht zu stehen, bei Schüssen auf die Knie fallen und streckte die Beine Richtung Torpfosten. 60 bis 70 Prozent der Tore fielen durch Flachschüsse. Roys Idee: „Man verhindert vermutlich viele, indem man ganz einfach auf die Knie geht. " Die Bewegungen erinnern an einen Schmetterling. Der Butterfly-Stil war zwar nicht neu, wurde aber durch Roy zum Standard. (SZ 14.10.2015)

Zum Beginn des Spiels und der Weiterführung nach Unterbrechungen wird ein Bully ausgeführt, der Einwurf des Pucks durch einen Schiedsrichter. Nach dem Oxford Dictionary of English (Stevenson 2010) ist das Wort seit dem späten 19. Jahrhundert belegt und bezeichnete im Eton football ein Gedränge, die genaue Herkunft ist nicht bekannt. Im deutschen Regelwerk wird die Aktion allerdings als Anspiel bezeichnet, im Englischen ist heute im Eishockey die Bezeichnung face-off im Regelwerk zu finden. Im Tschechischen ist zwar auch die Bezeichnung buly üblich, im Regelwerk heißt es jedoch offiziell vhazování ,Einwurf‘.

Die Entwicklung der deutschen Eishockeysprache ist sicher auch durch den dominierenden Fußball beeinflusst. So ist zu erklären, dass es im Regelbuch eben Tor statt Goal, Abseits statt Offside oder Verlängerung statt Overtime heißt. In den Medien, gerade bei Liveübertragungen von Spielen, ist aber auch Offside oder Overtime zu hören. Gleichzeitig sind Anglizismen, die durch den Fußball schon zum Kernwortschatz gehören, ganz selbstverständlich im Eishockey zu finden, so z. B. Pass und passen:

Noch in der 19. Minute glich der aufgerückte Moritz Müller nach einem maßgenauen Pass von Markus Eisenschmid aus. (HoWe 21.05.2021)

Zunächst behauptete Tom Kühnhackl stark im Zweikampf an der Bande den Puck, passte ihn in die Mitte, wo Stefan Loibl das Spielgerät perfekt traf und zum 1:0 (10.) erfolgreich war. (HoWe 24.05.2021)

Untersuchungen zur Geschichte des Eishockeys und seiner Sprache, beispielsweise in älteren Regelwerken und Zeitungsberichten, wären unter dem Gesichtspunkt der Verwendung von Anglizismen interessant. Im Führer durch die Sportsprache aus dem Jahre 1935 wird auf sehr romantisierende Weise auf den Ursprung des Eishockeys in Kanada und die Bedeutung dieses Landes für den Sport hingewiesen:

Wenn die breiten Burschen aus dem Land des Yukon, aus Toronto und Quebec, aus Montreal und Ottawa herüberkommen auf den alten Kontinent, dann geht ein ehrfürchtiges Gemurmel durch die Hallen. Sie spielen das größte ,Eishockey ' der Welt. Und in ihrer phantastischen Sportbekleidung, in der nie das Ahornblatt auf der Brust fehlt, stehen sie da wie die Götter des Spiels. (FDS:26)

Andererseits werden dort aber auch englischsprachige Ausdrücke als problematisch und überflüssig angesehen:

Seit einem halben Jahrhundert ist das umkämpfte Objekt die ,Scheibe . [...], Puck' heißt die Scheibe, und Stick sagte man einst für den Stock, überflüssige angelsächsische Bezeichnung. (FDS:26 f.). 
Die Verwendung des englisch-deutschen Wortpaares Puck vs. Scheibe wurde von mit Hilfe des bereits erwähnten Korpus untersucht (Winkler 2018:143 f.). In der Zählung in den Zeitungstexten wurde ebenfalls berücksichtigt, ob das Wort vom Autor des Artikels verwendet wurde oder ob die Äußerung eines Spielers oder Trainers zitiert wurde - in diesem Fall wäre die Nennung dem Sportjargon zuzuordnen. Bei der Zählung wurden auch Komposita, wie z. B. Scheibenverlust, berücksichtigt. Dabei kam Puck 34-mal vor (Jargon 2) und Scheibe 21-mal (Jargon 4). In den analysierten Zeitungsartikeln kommt also Puck häufiger vor, allerdings ist Scheibe ebenfalls oft belegt. In Bezug auf den Sportjargon ist sogar Scheibe häufiger zu finden, was dafür spricht, dass der deutsche Ausdruck keinen geringeren Fachlichkeitsgrad hat. Das Korpus ist nicht sehr groß, und daher sind diese Zahlen zur Verwendungshäufigkeit vorsichtig zu interpretieren. Aber Tendenzen sind doch zu erkennen, vor allem die Gegentendenz zur sonst beliebten Verwendung von Anglizismen ist bemerkenswert. Und es zeigt sich auch, dass in der gesprochenen Sprache ein Trend zum deutschen Wort Scheibe vorliegt. Dies wurde bestätigt durch die Analyse von 3 Fernsehübertragungen von Spielen. In diesen kam die deutsche Bezeichnung mit 116-mal sogar wesentlich häufiger vor als Puck mit 34-mal.

Ein Anglizismus, der aus dem Eishockey in die Sprache anderer Sportarten übernommen wurde, ist Check; Burkhardt (2006:62) führt „Ellenbogencheck“ als Übertragung in die Fußballsprache an. In der Bezeichnung von Strafen im Spiel im deutschen Regelbuch für das Eishockey (ReBuEi) kommt es in der Beschreibung von 7 Fouls vor: CHECK GEGEN DIE BANDE, CHECK VON HINTEN, CHECK GEGEN DEN KOPF ODER NACKEN, CHECK GEGEN DAS KNIE, CHECK MIT DEM STOCK, ELLBOGEN CHECK, CHECK MIT DEM KNIE (die Benennungen der Regeln sind im Regelbuch in Großbuchstaben geschrieben, diese Schreibung wird im vorliegenden Beitrag übernommen); in der englischen Originalfassung ist Check jedoch nur 3-mal zu finden. Es handelt sich also nicht um Übersetzungen, sondern um eine eigenständige Verwendung. Das spricht dafür, dass Check, zumindest in Bezug auf die Eishockeysprache, voll in den deutschen Wortschatz integriert ist.

Teilweise scheinen sich englische Ausdrücke in der Eishockeysprache zu verselbständigen. So lautet der Name der Regel 152 des Regelbuches KICKEN (in der englischen Version KICKING). Im Wortlaut der Regel wird auch schnell verständlich, dass es sich um den Vorgang handelt, der sehr einfach mit der deutschen Übersetzung treten bezeichnet werden könnte:

DEFINITION: Ein Spieler, der mit seinem Schlittschuh eine schwingende Bewegung ausführt, die gegen irgendeinen Körperteil eines Gegenspielers gerichtet ist. I. Ein Spieler der einen Gegenspieler tritt oder zu treten versucht, erhält eine Matchstrafe. (ReBuEi)

Im Fall von Regel 160 SLEW-FOOTING ist das nicht so einfach. Der Ausdruck ist für Außenstehende unverständlich, die entsprechende Erklärung der Regel ist allerdings anschaulich:

DEFINITION: Ein Spieler, der in gefährlicher Weise von hinten gegen den Fuß oder den Schlittschuh eines Gegenspielers tritt oder einen Gegenspieler zurückzieht, während er ihm gleichzeitig von hinten in die Füße kickt oder schlägt. (ReBuEi)

Problematisch bei diesem Anglizismus ist, dass es in der deutschen Sprache keine Entsprechung gibt, wie beispielsweise beim ähnlichen Vorgang BEINSTELLEN (ReBuEi, Regel 167). Dies und die Tatsache, dass diese Regel noch sehr neu ist und erstmals im hier zu Grunde gelegten Regelbuch von 2014 vorkommt, könnte dazu geführt haben, dass einfach das englische Wort übernommen wurde.

Spielt eine Mannschaft auf Grund einer Strafe für 2 oder mehr Minuten in Überzahl bzw. Unterzahl, werden dafür manchmal die Anglizismen Powerplay bzw. short-handed verwendet. Im bereits erwähnten Korpus (Winkler 2018:145 f.) wurde die Häufigkeit des Vorkommens von Powerplay resp. Überzahl untersucht. Sowohl bei den Zeitungsartikeln (Powerplay 14-mal, Überzahl 24-mal) als auch in den Fernsehübertragungen (Powerplay 16-mal, Überzahl 45-mal) kam das deutsche Wort häufiger vor, die englische Bezeichnung short-handed überhaupt nicht. In manchen Spielberichten, 
beispielsweise in Live-Tickern, sind die englischen Bezeichnungen Powerplay bzw. short-handed zur Kennzeichnung von Toren, die eine Mannschaft in Über- oder Unterzahl erzielt, üblich. Dass es für in diesen Spielsituationen erzielte Tore überhaupt eine Benennung gibt, liegt an der spielspezifischen Besonderheit, dass eine Mannschaft in Überzahl den Gegner unter enormen Druck setzen kann und die Wahrscheinlichkeit, ein Tor zu erzielen, deswegen wesentlich größer ist, als ein Gegentor zu bekommen. Im Vergleich dazu ist es beispielsweise im Handball nicht ungewöhnlich, dass eine Mannschaft in einer zweiminütigen Unterzahl Tore erzielt oder in Überzahl eben nicht. Vermutlich aus diesem Grund und da im Handball insgesamt mehr Tore erzielt werden, ist dort keine standardmäßige Benennung für die entsprechenden Tore üblich. Und wenn im Fußball von Powerplay geredet wird, dann ist damit etwas anderes gemeint - eine Mannschaft spielt sehr schnelle, kraftvolle Angriffe und übt starken Druck auf die gegnerische Mannschaft aus, wobei meist auch die Abwehrspieler sehr offensiv agieren:

„,Wir hatten uns für die zweite Halbzeit viel vorgenommen, hatten aber nie richtig Zugriff. Teilweise war es ein Powerplay von Bochum ", räumte Rechtsverteidiger Christopher Trimmel ein. (KiPr 4.12.2017, 65)

Es entwickelte sich ein minutenlanges Powerplay, in dem Hannover zwar zu zahlreichen Torannäherungen, aber zunächst nicht zu zwingenden Abschlüssen kam. (KiOn 18.12.16)

Wenn ein Spieler eine Zeitstrafe bekommt, dann verbringt er diese in der Box, manchmal auch Kühlbox oder Strafbox genannt:

Dann wurde es bitter für die Heimmannschaft, denn kurz nach Steckel wanderte auch Mitchell in die Box und diese doppelte Überzahl ließen sich die Haie nicht entgehen. (HoWe 24.01.2018)

Kurz darauf landete mit Sam Verelst noch ein weiterer Schwarz-Roter in der Box. (HoWe 24.11.2018)

Insgesamt saßen die Nürnberger viel zu oft in der Straf-Box, ganze 28 Strafminuten sammelten sie in der Partie. (HoWe 04.12.2016)

Im zweiten Abschnitt setzte sich die Flut an Strafen fort. Als Marcus Götz wegen übertriebener Härte in der Kühlbox saß, traf Steven Hanusch postwendend zum 1:0 für die Dresdner Eislöwen. [...] Keine 60 Sekunden später musste mit Philipp Preto erneut ein Falke in die Kühlbox. (HoWe 19.01.2021)

Die verbleibenden Mitspieler sind dann ebenfalls in der Box - sie sind in der Abwehr vor dem eigenen Tor als Viereck (als Box) ausgerichtet, da dies eine gute Verteidigung in Unterzahl ermöglicht:

Die Ice Tigers ließen den Puck gut zirkulieren, jedoch stand die Box der Adler gut und verhinderte so gute Chancen. (HoWe 13.02.2020)

Doch die Dänen standen eng in der Box und konnten ein Gegentor im ersten Spielabschnitt noch verhindern. (HoWe 13.01.2016)

Entsprechend wird das Spiel in Unterzahl auch als Boxplay bezeichnet:

Linz überzeugte in Spiel eins in Unter- und Überzahl: Es überstand alle sieben Box-Plays und scorte in zwei seiner vier Powerplay-Situationen. Die Special-Teams werden auch in Spiel 2 wieder entscheidend werden. (HoWe 05.03.2020)

Dagegen war das Boxplay bzw. Penaltykilling unterirdisch schlecht. (HoWe 20.05.2016)

Mit Penaltykilling haben wir eine weitere Bezeichnung, die vor allem darauf zielt, dass man in Unterzahl Gegentore verhindert, diese also unbeschadet übersteht. Da Penalty im Englischen allgemein für Strafe steht, finden wir diesen Ausdruck nicht nur bei den Zeitstrafen. Es gibt auch Strafschüsse, die dem Siebenmeter im Handball bzw. dem Elfmeter im Fußball entsprechen. Diese Bestrafung wird beispielsweise ausgesprochen, wenn ein angreifender Spieler in einer Break-Away-Situation (er hat den Puck unter Kontrolle, läuft allein auf den Torhüter zu, hat zwischen sich und dem Tor kei- 
nen gegnerischen Feldspieler und eine aussichtsreiche Torchance) gefoult wird. In diesem Fall sind die Formulierungen im Regelbuch allerdings nicht einheitlich - neben der häufigeren Verwendung von Penalty ist vereinzelt auch Strafschuss zu finden. Eine deutschsprachige Formulierung ist also möglich und nicht unüblich. Endet ein Spiel unentschieden und wird auch in der Verlängerung kein Tor erzielt, kommt es zum Penalty-Schießen, in der englischsprachigen Version des Regelbuches penalty-shot shootout. Die Bezeichnung Penalty scheint hier etwas irreführend, da es sich nicht um eine Bestrafung handelt, sie wird aber auch im Regelbuch für dieses Verfahren der Spielentscheidung verwendet.

Ein für Außenstehende nicht unbedingt verständlicher Anglizismus, der auch in der deutschen Version des Regelwerkes, in Stadiondurchsagen und in der Mediensprache verwendet wird, ist Icing. Dabei handelt es sich um das Hinausspielen des Pucks aus der eigenen Spielhälfte über die gegnerische Torlinie hinaus, also ohne dass es sich um einen Pass oder Torschuss handelt. Hierfür wäre die deutsche Bezeichnung ,unerlaubter Weitschuss bzw. Befreiungsschlag“, die im Sprachgebrauch in den Medien auch vorkommt, verständlicher.

Ein großer Unterschied zum Hand- und Fußball ist die Art der Fortbewegung. Auf dem Eis läuft man selbstverständlich nicht einfach, sondern fährt oder skatet:

„Er ist ein richtig guter Skater, aber, ehrlich gesagt, kannte ich ihn bis vor kurzem gar nicht“, sagte Routinier Felix Schütz über Tiffels, der selten lange zögert, wenn sich die Aussicht bietet, mit raumgreifenden Schritten weit in die gegnerische Zone vorzustoßen. (FAZ 04.05.2017)

Mit den Speedskatern Michaelis, Tiffels und Kahun, der nächste Saison in Chicago selbst in die NHLElite aufsteigen will, stehen ihm nun gleichaltrige Spielgefährten zur Verfügung. (SZ 04.05.2018)

Bei der Beschreibung von Taktik und Spielsituationen sind viele Anglizismen zu finden. Ein Ausdruck, der mittlerweile auch in der Fußballsprache gebräuchlich ist (s. o.), ist Forechecking, also eine Variante der Abwehr, bei der der angreifende Gegner bereits weit in dessen eigenen Spielhälfte attackiert und unter Druck gesetzt wird:

Also begannen die Wolfsburger, wie man sie kennt. Aggressives Forechecking, schnelles Umschaltspiel, stocktechnisch und im Zweikampf gut, einstudierte Laufwege [...] (HoWe 19.02.2017)

Die Tschechen agierten in einem sehr körperlichen Spiel aggressiv im Forecheck, aber Söderholms Mannschaft hielt dagegen. (HoWe 29.04.2021)

Vor dem kommenden Gegner Iserlohn warnt Co-Trainer Mike Pellegrims: „Iserlohn ist läuferisch extrem stark, sie haben offensiv viel Feuerpower und sind in Überzahl brandgefährlich. Sie spielen einen harten Forecheck. " (HoWe 29.03.2021)

Auch Turnover, der Verlust des Pucks, ist ein gebräuchlicher Ausdruck:

Die Finnen kamen über schnelle Turnover immer wieder gefährlich vor das Münchner Tor (HoWe 03.09.2018)

Tomas Larkin leitete ein Turnover auf Ronny Arendt ein, dessen Pass vors Tor verwertete Mannheims Kapitän Markus Kink, der zu den Spielern der Adler gehört, der effektiv in Play-off-Form ist. (HoWe 21.03.2017)

Geht es nach Dennis Seidenberg, dem erfahrenen NHL-Spieler von den New York Islanders, dann leistet sich Deutschland viel zu viele einfache Scheibenverluste in der neutralen Zone oder an der eigenen blauen Linie. ,Wir müssen einfaches Eishockey spielen: Scheibe tief, forechecken und Turnover erzwingen. Wir dürfen nicht schlampig sein und müssen besser auf die Scheibe aufpassen, dann werden wir auch Erfolg haben. " (SP, 10.05.2017)

Das Ziel ist in allen drei Sportarten gleich: der Sieg. Dazu muss man Tore erzielen oder seine Mitspieler dabei mit guten Zuspielen, den Assists, unterstützen. Für Tore und Assists gibt es dann Scorerpunkte: 
Der gebürtige Kölner hat in 71 Spielen 43 Tore und 67 Vorlagen erzielt und kommt damit auf 110 Scorerpunkte. (HoWe 27.05.2020)

Leon Draisaitl belegt mit 61 Scorerpunkten (22 Tore, 39 Vorlagen) weiterhin den zweiten Platz in der Scorerwertung hinter Connor McDavid, der 68 Punkte (23 Tore, 45 Assists) aufweist. (HoWe 08.04.2021)

Die Edmonton Oilers überrollten förmlich die Ottawa Senators, die der geballten Offensivkraft von Leon Draisaitl (drei Tore, zwei Assists) und Connor McDavid und Verteidiger Tyson Barrie (je drei Punkte) nichts entgegenzusetzen hatten. (HoWe 11.03.2021)

Dass die Assists mit einem Punkt bewertet und auf diese Weise in der offiziellen Wertung den Toren gleichgestellt werden, ist eine Besonderheit des Eishockeys gegenüber Handball und Fußball. Im Fußball wird eine entsprechende Punktewertung mittlerweile allerdings ebenfalls in vielen Statistiken, beispielsweise im Kicker, angeboten, und man findet entsprechende Hinweise auch in Spielberichten:

Dabei müssen die Gastgeber allerdings auf den aktuell besten Scorer der Liga, Baris Atik (sechs Tore/acht Assists), aufgrund der fünften Gelben Karte verzichten. (KiPr 21.10.2021, 48)

Im Handball gibt es ebenfalls eine Zählung der Assists, allerdings ist eine Wertung nach Scorerpunkten nicht üblich und nur vereinzelt anzutreffen:

Mit neun Toren und ebenso vielen Assists schwang sich der Spielmacher des Meisters zum entscheidenden Spieler des Spitzenspiels auf. (HaWo 04.10.2017, 18)

Beim DHB-Team war Alina Grijseels die überragende Spielerin. Die Dortmunderin beendete das Turnier mit 35/9 Toren auf Rang 15 der Torschützenliste und ihre 33 Assists bringen ihr Position 14. Das führt dann allerdings bei der Scorerwertung mit 68 Punkten zu Platz 9. (HaWd 20.12.2021)

Die meisten Punkte sammeln dabei im Eishockey die Angriffsspieler - die Flügelspieler an den Außenseiten und der Center (auch deutsch Mittelstürmer) in der Mitte:

Sturm nominierte Draisaitl nach einem kurzen Vier-Augen-Gespräch sogleich für die Startformation als Center der ersten Reihe, unterstützt von Brooks Macek (München) und Matthias Plachta (Mannheim) auf den Flügeln. (FAZ, 15.05.2017)

Für das Erzielen von Toren gibt es verschiedene Schussmöglichkeiten. Beim One-Timer handelt es sich um einen Schuss, bei dem nach einem Pass der Puck direkt geschossen wird, ohne ihn vorher anzunehmen und zu kontrollieren:

In doppelter und einfacher Überzahl legte die EGDL anschließend nach: RJ Reed hämmerte die Scheibe als One-Timer in den Winkel (11.). (HoWe 15.02.2020)

Die erste gute Chance hatte Bremerhaven in Minute 44, doch Rynnäs rettete, ebenso wie Sekunden später nach einem Onetimer von Dietz. (HoWe 12.12.2019)

Je nachdem, wie dabei der Schläger gehalten wird, gibt es wieder Unterschiede. Bei einem Rückhandschuss spricht man auch von einem Backhander:

Das Tor erzielte aber auf der anderen Seite Frömter, der nach einem Konter Thiem mit einem Backhander überwand. (HoWe 08.12.2019)

Der ungebrochene Offensivdrang sollte den Schwaben jedoch wenig später zum Verhängnis werden, denn Philipp Schlager setzte in der 14. Minute zum Konter an und vollendete diesen mit einem Backhander ins Kreuzeck. (HoWe 24.08.2014)

Wird ein Schuss von der Blauen Linie, also dem Anfang der Angriffszone abgegeben, handelt es sich um einen Blueliner: 
Marco Nowak besorgte per Blueliner die Düsseldorfer Führung. (HoWe 17.12.2018)

Die deutsche Mannschaft setzte die Eidgenossen früh unter Druck und hatte durch einen Blueliner von Simon Gnyp (3.) und einem Torschuss von Dominik Bokk aus dem Slot die ersten guten Gelegenheiten. (HoWe 13.12.2019)

Diese Schüsse werden von den Verteidigern abgegeben, die sich, vor allem im Powerplay, an der blauen Linie aufhalten, daher werden auch sie Blueliner genannt:

Vor allem im Powerplay weiß der Blueliner seinen knallharten Torschuss einzusetzen. (HoWe 28.02.2021)

42 Tore und 136 Assists stehen dabei für den 31-jährigen Blueliner in der Statistik. (HoWe 08.03.2020)

Offensiv setzt Lamb als guter Aufbauspieler und schussstarker Blueliner immer wieder Akzente. (HoWe 04.02.2017)

Dieser Blueliner ist eine Personenbezeichnung auf er. Im Unterschied zu Keeper (s. o.) ist diese jedoch nicht von einem Verb abgeleitet, sondern von einem Substantiv. Für Eisenberg (2011:262) handelt es sich bei solchen Ableitungen nicht um ,echte Nomina Agentis“, und es gibt für diese Wortbildungen „kein produktives Analogon in der Kerngrammatik“. Auch der Blueliner als Schuss, der von der Blauen Linie abgegeben wird, ist eine Ableitung auf er. Jedoch handelt es sich hierbei um eine Metonymie, bei der von einem besonderen Umstand des Schusses ausgegangen wird. Ebenso ist es beim Backhander. Ein analoges Wortbildungsmuster gibt es im Deutschen allerdings nicht. Trotz dieser Unterschiede zu Keeper und analog gebildeten Substantiven sind diese er-Sustantive hinsichtlich Flexion und Wortbildungsmöglichkeiten ebenso ins Deutsche integriert wie diese.

Direkt vor dem Tor befindet sich der Slot. Dabei handelt es sich um ein virtuelles Viereck, dessen Grundseite das Tor markiert und das sich bis auf die Höhe der Bullypunkte erstreckt:

Die Zone direkt vor dem Tor ist im Eishockey jene, die angreifenden Stürmern verlässlich blaue Flecken beschert. Wer sich im Slot-so wird dieses Areal in der Eishockeysprache genannt-aufhält, der wird mit sehr hoher Wahrscheinlichkeit von den gegnerischen Verteidigern recht unsanft bearbeitet. Der Slot ist so hart umkämpft, weil dort die Chance am höchsten ist, einen Schuss abzufälschen, einen Abpraller zu bekommen oder dem gegnerischen Torhüter die Sicht zu nehmen. Man muss es mögen, sich in dieser umkämpften Zone aufzuhalten. (SZ 29.12.2019)

Er bekam einen unglücklichen Abpraller von Brückmann im Slot direkt auf den Schläger und traf. (HoWe 04.02.2021)

Francois Methot passte in den Slot, wo Marcus Kink lauerte und im zweiten Versuch die Scheibe im Tor von Storr unterbrachte. (HoWe 16.11.2007)

Tiffels zieht aus dem Slot ab, der Puck rauscht aber links neben den Pfosten. (SP 23.05.2019)

Diese Zone ist nicht zuletzt gefährlich wegen der Abpraller, die einen Nachschuss ermöglichen, der Rebounds:

Als Denis Reul zu Beginn des Schlussdrittels per Schlagschuss nach Rebound zum 2:1 erfolgreich war (42.), schien sich die Überlegenheit, die sich in der Schussstatistik (34:16 für Deutschland) ausdrückte, auch in Tore umzusetzen. (HoWe 16.05.2016)

Der Ausgang des Spiels hatte aber vor allem mit dem guten Auftritt der Gastgeber zu tun, die in der siebten Minute ein Überzahlspiel per Rebound von Mitch Wahl zur 1:0-Führung ausnutzten. (HoWe 10.12.2020)

In der sechsten Minute war eine Überzahlsituation gerade zu Ende gegangen, als Marcel Goc sich seinen eigenen Rebound schnappte und aus der Drehung zur Adler-Führung traf. (HoWe 26.04.2019) 
Im nächsten Beispiel geht es um ein garbage goal. Dabei handelt es sich um ein Tor, das nicht schön, nicht klar herausgespielt ist. Es ist in diesem Fall kein eindeutiger Schuss, sondern mit wurstelt wird im folgenden Beispiel passend umschrieben, dass der Puck im Gewirr glücklich ins Tor befördert werden konnte:

Schütz selbst war bei diesem Turnier noch nicht viel gelungen, er hatte, wie er hinterher erzählte, am Morgen noch seinen Schläger in eine Mülltonne in der Kabine gesteckt, ein Ritual in der Hoffnung auf ein ,garbage goal“", ein dreckiges Tor. 33 Sekunden vor der Schlusssirene stand Schütz dann am zweiten Pfosten und wurstelte die Scheibe zum Ausgleich ins Netz. (SZ 17.05.2017)

Liegt eine Mannschaft wenige Minuten vor dem Ende mit ein oder zwei Toren zurück, verzichten Trainer oft auf den Torhüter zugunsten eines zusätzlichen Feldspielers. Auf diese Weise kann man zwar im Angriff mehr Druck erzeugen, es erhöht aber auch das Risiko, ein Gegentor ins leere Tor empty net - zu bekommen:

Das Ergebnis lässt Schlimmes vermuten, doch wenn man sich die einzelnen Drittelergebnisse (1:1; 0:0; 5:1) ansieht und berücksichtigt, dass die letzten beiden Tore Empty Net Goals waren, relativiert sich alles wieder ein wenig. (HoWe 13.11.2004)

Simon Sezemsky (38., Überzahl) gelang das einzige Tor für die Auswahl des Deutschen EishockeyBundes gegen den Weltranglistenfünften, der sich den Erfolg durch zwei späte Empty-Net-Tore sicherte. (HoWe 29.04.2021)

Erst erzielte Connor McDavid das 3:2 (54.) und exakt eine Sekunde vor Schluss markierte Draisaitl per Empty Netter das 4:2 und vervollständigte so seinen Hattrick. (HoWe 08.04.2021)

Endet ein Spiel in den regulären 60 Minuten Spielzeit unentschieden, kommt es zu Verlängerung. Diese wird im Modus Sudden Death (im Fußball früher zeitweise als Golden Goal üblich) gespielt, also das erste erzielte Tor bringt den Sieg. Die Entscheidung zur Meisterschaft wird in den Playoffs entschieden. Entsprechungen wie Ausscheidungsrunde oder Ausscheidungswettkampf sind im deutschen Sprachgebrauch nicht etabliert. Die Playoffs werden im Best-of-Seven-Modus gespielt, also diejenige Mannschaft, die in 4 Spielen siegreich ist, gewinnt auch die Serie.

Im Eishockey sind deutlich mehr Anglizismen zu finden als im Fußball. Viele sind auch klar als solche zu erkennen und gehören nicht zum Kernwortschatz des Deutschen (Icing, Scorer, Shootout, Face-off). Dieses häufige Vorkommen von Anglizismen ist sicher durch den großen nordamerikanischen Einfluss bei der Entwicklung dieser Sportart in Deutschland, vor allem nach dem 2. Weltkrieg, zu erklären. Dieser Einfluss ist in der Struktur und an der Spielweise in der Liga erkennbar. Nicht zuletzt liegt das auch an den vielen nordamerikanischen und anderen ausländischen Spielern und Trainern, deren Wirken zur Folge hat, dass die Arbeitssprache in den Mannschaften meist Englisch ist. Der Anteil ausländischer Spieler ist mit 47,6 \% sehr groß, höher ist er nur in der englischen Liga (Stand vom 28.05.2021) (URL 1). Davon kommen 76,8 \% aus Kanada oder den USA (das sind 32,3 $\%$ aller Spieler insgesamt). Im Vergleich dazu liegt beispielsweise der Ausländeranteil in der Extraliga in Tschechien bei 16,2\% (URL 2). Davon sind allerdings 49,2\% Slowaken, die von den meisten Tschechen nicht als Ausländer im engeren Sinne betrachtet werden und für die das Tschechische auch keine Sprachbarriere darstellt, sodass eine andere Arbeitssprache in den Mannschaften nötig wäre.

\section{Shooter in der Crunch-Time mit Buzzer-Beater - Handball}

Im Vergleich zu den anderen beiden Sportarten finden sich im Handball die wenigsten Anglizismen; so ist beispielsweise ein Turnover einfach ein Ballverlust und ein Reebound ein Abpraller. Zwar wird nicht selten von einem Konter gesprochen, aber der handballspezifische Ausdruck ist Tempogegenstoß. Im Führer durch die deutsche Sportsprache aus dem Jahre 1935 wurde, mit den seinerzeit üblichen nationalistischen Untertönen, festgestellt: 
Handball ist ein deutsches Spiel. Daher ist auch seine Sprache rein deutsch. Die Sprache der Handballplätze ist ein Fußballjargon ohne englische Anklänge. (FDS:17)

Seit dieser Zeit hat sich aber nicht nur der Handballsport sehr gewandelt, nicht zuletzt wird er seit Jahrzehnten in Hallen gespielt und nicht mehr auf dem großen Feld im Freien. Auch die Sprache hat sich verändert, und es gibt nun ebenfalls englische Ausdrücke. Da Pass und passen bereits zum Kernwortschatz des Deutschen gehören, sind sie im Handball nicht mehr wegzudenken, auch entsprechende Komposita sind üblich:

Wird von der angreifenden Mannschaft nach maximal 6 Pässen kein Torwurf ausgeführt, wird auf Freiwurf gegen die ballbesitzende Mannschaft entschieden. (ReBuHa 7:12)

Aus der Wurfauslage mit Blick Richtung Tor wird dann zum nächsten ins Zentrum anlaufenden Spieler gepasst. (HaTr 16)

Eine hohe Passgeschwindigkeit ist im Spielaufbau notwendig. Ein probates Mittel sind dabei auch Expresspässe, d.h. Pässe, bei denen Positionen übersprungen werden (z. B. Außen zu RM oder RL zu RR). Expresspässe haben das Ziel, den Ball schneller zu passen, als die Abwehr verschieben kann. (HaTr 15)

Eine besondere Variante sind No-Look-Pässe, bei denen sich der Spieler, zu dem der Ball gespielt wird, nicht im direkten Sichtfeld befindet:

Bei ihrer intelligenten Spielsteuerung setzt sie nicht nur ihre Mitspielerinnen toll in Szene, sondern glänzt auch selbst mit sehenswerten No-Look-Pässen. (HaWd 26.01.2020)

Sonderbeifall gab es dann für den zwölften THC-Treffer: Sakia Lang hatte mit einem No-Look-Rückhandpass Meike Schmelzer am Kreis freigespielt. (HaWd 11.02.2018)

Diese Pässe und den dazugehörigen Anglizismus gibt es auch im Fußball und im Eishockey:

Der Mannheimer nahm aus vollem Lauf einen No-Look-Pass quer durch die kanadische Zone an, schaute kurz, wo Poulin stand, und zog unhaltbar unter die Querlatte zum 2:0 ab (24.). FAZ 23.02.2018

Auch die Fouls kommen regelmäßig vor und sind daher im Regelbuch und in Handbüchern zu finden:

Die vielen Fehler und Fouls sind verantwortlich für viel zu häufige Unterbrechungen. (HaTr 13)

Erzielt der gefoulte Spieler oder ein Mitspieler noch vor der Spielunterbrechung ein Tor, entfällt der

7-m-Wurf. (ReBuHa 8:10)

Dass im Handball auch Keeper im Tor stehen, dass Spieler sich durchtanken und dass Assists wichtig für Tore sind, wurde bereits ausgeführt. Es sind aber auch weitere neue Anglizismen zu finden, die klar als solche erkennbar sind. So werden Spieler, die vor allem aus dem Rückraum sehr wurfstark sind, als Shooter bezeichnet, wobei auch Zusammensetzungen üblich sind:

„Emily Bölk ist eine Shooterin, die darüber hinaus mit einem hohen Maß an Spielfähigkeit ausgestattet ist. Und das macht sie so wertvoll und begehrt", schwärmt auch Trainer Dirk Leun (HaWo 07.03.2017, 33)

In dieser Phase fasst sich Göppingens Rückraumshooter Jens Schöngarth ein Herz und erzielte aus der Distanz zwei tolle Treffer. (HaWo 24.10.2017, 21)

Rückraumspieler Michael Damgaard entpuppt sich mehr und mehr als der Top-Shooter im Team des SC Magdeburg. (HaWo 13.06.2017, 25)

Wenn eine Mannschaft in der Abwehr den Ball erobert und in den Angriff übergehen kann, der Ball also der angreifenden Mannschaft gestohlen wird, handelt es sich um einen Steal: 
Endgültig entschieden war die Partie, als Rechtsaußen Florian von Gruchalla ein Steal gelang und er zum 21:25 (57.) traf. (HaWo 10.10.2017, 19)

Weiß verleitete die Lemgoer zu einem technischen Fehler und verwandelte den Steal zum 24:24. (HaWo 23.05.2017, 20)

Häufig werden Spiele erst in den letzten Minuten entschieden, und es ist ganz normal, dass selbst zwei Minuten oder selbst 30 Sekunden vor dem Ende noch alles offen ist. Diese Schlussphase ist dann besonders hart umkämpft - es ist die Crunchtime:

Bennet Wiegert: „Haben es in der Crunchtime für uns entscheiden können“ (HaWd 16.12.2019)

Rolf Brack (Trainer Frisch Auf Göppingen): , Wir hatten heute die richtige kämpferische Grundeinstellungen und konnten dann auch mit etwas Glück das Spiel in der Crunchtime für uns entscheiden. “ (HaWo 13.02.2018, 17)

Michael Haaß stellte in der Crunch-Time auf eine 5:1-Abwehr um, doch die Gäste aus Mannheim nutzten die sich bietenden Räume clever, um auf 18:22 zu erhöhen. (HaWd 26.10.2020)

Die Entscheidung fällt dann manchmal in den letzten Sekunden, es entscheidet ein letzter Torwurf kurz vor dem Ertönen der Schlusssirene - ein Buzzer-Beater:

Mit einem Buzzer-Beater in buchstäblich letzter Sekunde sicherte sich der TuSEM Essen beim 26:26 (12:14) gegen die TSV Hannover-Burgdorf einen überraschenden Zähler. (HaWd 30.11.2020)

Sebastian Heymann sorgte per Buzzer-Beater für den Siegtreffer. (HaWd 06.03.2021)

Der Aufsteiger sicherte sich mit einem Buzzer-Beater von Lucas Firnhaber einen Punkt gegen die TSV Hannover-Burgdorf. (HaWd 29.11.2020)

Nach einem Acht-Tore-Vorsprung bringt erst ein Buzzer-Beater die Entscheidung: Frisch Auf Göppingen und die Rhein-Neckar Löwen lieferten sich beim 32:31 (17:15) einen unglaublichen Schlagabtausch. (HaWd 06.03.2021)

Bei Shooter, Steal, Crunchtime und Buzzer-Beater handelt es sich um Ausdrücke, die aus der Sprache des Basketballs entnommen sind, die (wie die Eishockeysprache) durch nordamerikanische Einflüsse geprägt und reich an Anglizismen ist. In Anlehnung an den Basketball wird im deutschen Handball auch immer wieder über die Einführung einer Shot clock zur Regelung des Zeitspiels diskutiert. Und die Auszeiten von einer Minute, die von den Mannschaften genommen werden können, heißen sogar im deutschen Regelbuch Team-Time-out (ReBuHa 2:8).

Der Höhepunkt der großen Pokalwettbewerbe im europäischen und deutschen Handball ist das Final Four. Dieses findet jeweils an einem Wochenende statt, am Sonnabend gibt es die Halbfinalspiele, am Sonntag dann das Spiel um den 3. Platz und das Finale:

Das Final Four um den DHB-Pokal 2020 wird Anfang Juni ausgetragen, das gab die HBL am Mittwoch bekannt. (KiOn 28.04.2021)

Der Bundesliga-Aufsteiger ist die große Überraschung beim Final4 und wohl der absolute Außenseiter (HaWo 11.05.2021, 39)

In den letzten Jahrzehnten ist eine leichte Tendenz zur Nutzung von Anglizismen zu beobachten. Allerdings betreffen sie - anders als im Fußball oder Eishockey - nicht wichtige taktische Elemente des Spiels. Auch englische Bezeichnungen für Spielerpositionen - wie im Tschechischen pivot ,Dreh- und Angelpunkt' für Kreismitte - sind in der deutschen Handballsprache nicht üblich. Zur Fachsprache im engeren Sinne gehören lediglich Team-Time-out, Assist, Pass und Foul (wobei zumindest die letzten beiden als Entlehnungen aus der Fußballsprache anzusehen sind). Die anderen englischen Ausdrücke sind eher zum Sportjargon oder zur Mediensprache zu rechnen.

Trotz dieser Entwicklungen ist die Sprache des Handballs weitgehend Deutsch. Das betrifft auch die Arbeitssprache in den Mannschaften. Der Anteil an ausländischen Spieler in der Liga ist 
mit ca. $40 \%$ zwar deutlich geringer als im Eishockey. In den Spitzenmannschaften liegt er jedoch mit um die $70 \%$ erheblich höher, wobei Skandinavier und Spieler aus den Staaten des ehemaligen Jugoslawien den größten Anteil stellen (URL 3). Auch die Herkunft der Trainer - gegenwärtig (Saison 2020/21) sind es fünf in der Liga (drei Tschechen, ein Isländer und ein Spanier) und ein isländischer Bundestrainer - hat keinen Einfluss auf die Sprache. Einen personell bedingten Einfluss des Englischen gibt es also im Handball nicht. Eher das Gegenteil ist zu beobachten - ein Einfluss des Deutschen auf den Handball in anderen Ländern. Das belegen die Aussagen des dänischen Nationaltorhüters und Welthandballers von 2019 Niklas Landin über die sprachliche Realität in der dänischen Nationalmannschaft und bei Paris Saint-Germain sowie über einen kroatischen Trainer deutlich:

\begin{abstract}
Die deutsche Sprache ist jedoch nicht nur in Landins Heimaturlauben sehr präsent. Im Kreise der Nationalmannschaft schleicht sich während der Großturniere im dänischen Umfeld ebenfalls Deutsch ein, wie Niklas Landin verrät: ,Auch, wenn ich da im Tor stehe, rede ich meistens auf Deutsch, das bin ich bei den Spielen einfach so gewohnt", erzählt der Kapitän der Dänen und des THW Kiel, und verdeutlicht das direkt an einem Beispiel aus seinem Arbeitsalltag zwischen den Pfosten: ,Wenn ein Gegenspieler einläuft, dann sage ich das auf Deutsch und nicht auf Dänisch, da überlege ich gar nicht erst. Ich könnte nicht sagen, wann ich dafür das letzte Mal Dänisch benutzt habe. "Anderswo würde das vielleicht zu Verständigungsproblemen führen, nicht aber bei den Dänen: „,Die meisten meiner Teamkollegen aus der Nationalmannschaft spielen ja aktuell in Deutschland oder haben hier mal gespielt. Magnus spielt hier in Kiel, ein Großteil der anderen in Flensburg, Mikkel Hansen wurde bei Paris Saint-Germain von Noka Serdarusic trainiert, der dort ja auch nur Deutsch gesprochen hat. Ich glaube schon, dass wir auch in der dänischen Nationalmannschaft alles auf Deutsch machen könnten “, ist Landin sicher. (URL 4)
\end{abstract}

Und bei den Olympischen Spielen 2021 sorgte ein Video nach dem Spiel Frankreich gegen Norwegen für Verwunderung. Kurz nach dem Ende brachte der französische Spieler Nikola Karabatić gegenüber dem norwegischen Co-Trainer Børge Lund seinen Unmut zum Ausdruck - in deutscher Sprache:
„Zwanzig Sekunden vor dem Ende nimmst Du Time-Out? Das ist Verarschung“, so Nikola Kara- batic in Richtung des früheren Mitspielers, der als Co-Trainer für Norwegen im Einsatz ist. (HaWd 01.08.2021)

Nun ist Deutsch zwar keine Lingua franca im internationalen Handball, hat aber doch eine herausgehobene Stellung.

\title{
5. Finish
}

Das Vorkommen von Anglizismen ist in den drei Sportarten sehr unterschiedlich - das ist nicht überraschend, deshalb wurden sie ja ausgewählt. Im Eishockey sind englische Ausdrücke am stärksten präsent, im Handball ist der englischsprachige Einfluss am geringsten. Der Fußball spielt bei der Entwicklung von Anglizismen eine große Rolle, da er sich auf Grund seiner Popularität auf die Sportsprache in Deutschland überhaupt auswirkte und auswirkt. Viele der vorkommenden Anglizismen gehören inzwischen zum Kernwortschatz des Deutschen, insbesondere viele Ein- und kurze Zweisilber (Foul, Pass, Konter). Diese sind so stark integriert, dass sie sich von Fremd- zu Lehnwörtern entwickeln (wie z. B. Sport, Training, Sprint). Auch die Wortbildung hat Einfluss auf die Integration von Anglizismen. Am Beispiel von Substantiven auf er wurde gezeigt, dass insbesondere die Bildung von Personenbezeichnungen, die aus Verben gebildet werden, dem Deutschen entspricht. Sie sind daher perfekt an das morphologische System angepasst. Es ist mit Eisenberg (2011:27) davon auszugehen, ,dass Form und grammatisches Verhalten von Wörtern für Normalsprecherinnen und Normalsprecher bei weitem die wichtigsten Anzeichen dafür liefern, ob ein Wort für sie fremd ist oder nicht“". 
Ein Blick in die Geschichte der Sportsprache zeigt Entwicklungstendenzen auf. Anglizismen, die bereits in der Frühzeit des Fußballs in Deutschland den Bemühungen um eine Verdeutschung widerstanden, sind heute integriert und fanden auch Eingang in die Fachsprache andere Sportarten. Andererseits sind etablierte Verdeutschungen ebenso im anglizismenreichen Eishockey gebräuchlich (Abseits). Der Einfluss von fachlichen Innovationen auf den Wortschatz zeigt sich sehr deutlich in der modernen Fußballsprache (Pressing, Forechecking, One-Touch-Fußball, Chip-Ball). Diese gehören ganz sicher nicht zum Kernwortschatz des Deutschen. Sie werden von Durchschnittssprechern als Anglizismus und als Fachwort identifiziert. Bei Fachleuten der Sportart (vom Trainer bis zum fachlich interessierten Fan) ist das selbstverständlich anders. Für sie gehören diese Fachwörter zum Alltagswortschatz und werden nicht mehr unbedingt als fremdes Wort wahrgenommen. Daher ist es ,nicht möglich, die Form eines Wortes zum absoluten Kriterium für Fremdheit zu machen und dann unter Fremdheit etwas für alle Sprecher in derselben Weise Gültiges zu verstehen“ (Eisenberg 2011:26). Noch deutlicher wird das beim weniger bekannten Eishockey, so nehmen Durchschnittssprecher Icing nicht nur als Fremdwort wahr, sondern verstehen es auch nicht.

Neben der Herkunft solcher Fachwörter spielt ebenso der Fachlichkeitsgrad eine Rolle. Für die Entwicklung der Sportsprache im Fußball und Eishockey gilt auch die von Eisenberg (2011:149) formulierte „Faustregel, dass ein Fachwortschatz umso mehr Fremdwörter enthält, je systematischer er ausgebaut wird“. Den Zusammenhang von Anglizismenverwendung und Fachlichkeit weisen Biskupová/Pišl (2019) ebenfalls für die Fachsprache der Wirtschaft nach.

Im Handball hingegen ist die Situation anders: Die vorkommenden Anglizismen prägen nicht den Fachwortschatz im engeren Sinne (wie im Fußball oder Eishockey), sie betreffen nicht die Spieltaktik oder wichtige technische Aspekte. Darüber hinaus sind die neueren Anglizismen nicht originär im Handball entstanden, sondern aus anderen Sportarten entlehnt.

Für den Status von Anglizismen und das Prestige des Englischen in der Sportsprache sind auch soziale Faktoren mitentscheidend. Es ist immer zu hinterfragen,

„aus welchen Gründen und mit welchen Wirkungen es zu Entlehnungen kommt [...]. Die Art des Sprachkontaktes, die beteiligten Sprechergruppen, die Verwendung der vorhandenen und der neu aufgenommenen Wörter lassen teilweise direkte Rückschlüsse oder doch plausible Annahmen darüber zu, wie sich der Fremdwortschatz entwickelt hat und was wir heute davon vorfinden" (Eisenberg 2011:4).

Im Eishockey ist sicher die Bedeutung Nordamerikas, insbesondere die Übermacht der NHL, die als Vorbild und Maßstab gilt, entscheidend für den starken englischen Einfluss. Im Fußball wird zwar auch die englische Premier League als stärkste Liga der Welt angesehen, sie ist aber bei weitem nicht so dominant. Sprachlich ist ihr Einfluss dennoch nicht zu unterschätzen. Im Handball dagegen ist die deutsche Liga die stärkste der Welt, daher ist sie auch attraktiv für ausländische Spieler und ein positiver Faktor für die deutsche Sprache.

\section{Literaturverzeichnis}

\section{Primärliteratur:}

FDS: Gloning, Thomas (2015): Der „Führer durch die Sportsprache“ (1935). Nachdruck und sprachliche Kommentierung. Gießener Elektronische Bibliothek. Zugänglich unter: http://geb. uni-giessen.de/geb/frontdoor.php?source_opus=11824\&la=de [28.05.2021]

FuKTTC: Bisanz, Gero / Gerisch, Gunnar (2013): Fußball. Kondition - Technik - Taktik und Coaching. Aachen, 2. überarbeitete Auflage.

HaWd: handball-world, www.handball-world.de.

HaWo: Handballwoche, Printausgabe.

HaTr: handballtraining 02/2009, Münster.

HoWe: Hockeyweb, www.hockeyweb.de.

KiOn: Kicker, www.kicker.de. 
KiPr: Kicker, Printausgabe.

KuAu: KoсH, Konrad (1903): Deutsche Kunstausdrücke des Fußballspieles. In: Zeitschrift des Allgemeinen Deutschen Sprachvereins 18, Nr. 6, Sp. 169-172. Zugänglich unter: https://www. deutschestextarchiv.de/book/show/koch_kunstausdruecke_1903 [28.05.2021]

FAZ: Frankfurter Allgemeine Zeitung, www.faz.net.

ReBuEi: Official Rule Book 2014-2018, herausgegeben von der International Ice Hockey Federation und die deutsche Version Offizielles Regelbuch 2014-2018.

ReBuFu: Laws of the Game 2017/18, herausgegeben von The International Football Association Board und die deutsche Version Spielregeln 2017/18.

ReBuHa: Rules of the Game, herausgegeben von der International Handball Federation und die deutsche Version Internationale Hallenhandball-Regeln, 2016.

SP: SPIEGEL, www.spiegel.de.

SZ: Süddeutsche Zeitung, www.sueddeutsche.de.

\section{Sekundärliteratur:}

Aмmon, Ulrich (1995): Die deutsche Sprache in Deutschland, Österreich und der Schweiz. Das Problem der nationalen Varietäten. Berlin; New York.

Biermann, Christoph (2019): Eine kurze Geschichte des modernen Fußballs. In: 11 Freunde \#209. April 2019, S. 25-34.

BiskuPOvÁ, Veronika / PišL, Milan (2019): Anglizismen in der Rubrik Wirtschaft der deutschen Tagespresse: Hilfe für Übersetzer oder Hürde für Leser? In: Acta Facultatis Philosophicae Universitatis Ostraviensis Studia Germanistica, Nr. 24, S. 19-30. Zugänglich unter: https://dokumenty. osu.cz/ff/journals/studiagermanistica/2019-24/SG_24_2_Biskupova_Pisl.pdf [28.05.2021]

Born, Joachim (2009): Vom Stufenbarren in die Halfpipe. Die deutsche Sportsprache im historischen Wandel. In: Burkhardt, Armin / Schlobinski, Peter (Hrsg.): Flickflack, Foul und Tsukahara: der Sport und seine Sprache. Mannheim; Leipzig; Wien; Zürich, S. 11-33.

BRÖKER, Frank (2013): Eishockey in Deutschland. Nichts für schwache Nerven. Meine.

BRÖKER, Frank (2016): Eishockey - Das Spiel, seine Regeln und ein Schuss übertriebene Härte. Meine.

BuRKHARDT, Armin (2006): Sprache und Fußball. In: Muttersprache, Nr. 1/2006, S. 53-73.

BuRKHARDT, Armin (2006a): Wörterbuch der Fußballsprache. Göttingen.

BurkHARDT, Armin (2008): Wenn das Leder im Kasten klingelt ... der deutsche Fußball und seine Sprache. In: GutJahr, Jacqueline / Yu, Xuemei (Hrsg.): Aspekte der Studienvorbereitung und Studienbegleitung. München, S. 68-87.

Burkhard, Armin (2008a): Der deutsche Fußball und seine Sprache. Nebst Kurzporträt eines Wörterbuchprojekts. In: KeMPER, Dirk / BäcKer, Iris (Hrsg.): Deutsch-russische Germanistik. Ergebnisse, Perspektiven und Desiderate der Zusammenarbeit. Moskau: Stimmen der slavischen Kultur (= Thomas Mann-Lehrstuhl an der RGGU Moskau, Institut für deutsch-russische Literatur- und Kulturbeziehungen, Schriftenreihe, Band 1), S. 223-244.

Burkhard, Armin (2008b): Anglizismen in der Fußballsprache. Eine historische und kontrastive Betrachtung. In: Der Sprachdienst, Heft 2, S. 57-69.

BurKhard, Armin (2015): Konrad Koch und Olga Eckardt - zwei Meilensteine in der Geschichte der deutschen Fußballsprache und ihrer Dokumentation. In: BoRn, Joachim / GLONING, Thomas (Hrsg.): Sport, Sprache, Kommunikation, Medien. Interdisziplinäre Perspektiven. Gießener Elektronische Bibliothek, S. 93-132. http://geb.uni-giessen.de/geb/frontdoor.php?source_opus $=11823 \&$ la $=$ de $[28.05 .2021]$

BurkHARDT, Armin (2021): Fußballsprache(n). Ein Beitrag zur Kontrastiven Onomasiologie. In: Ladilova, Anna / Leschzyk, Dinah / Müller, Katharina / Schweitzer, Nicolas / Seiler, Falk (Hrsg.) Bornistik. Sprach- und kulturwissenschaftliche Perspektiven auf die Romania und die Welt. Giessen University Library Publications, S. 41-58. http:/geb.uni-giessen.de/geb/volltexte/2021/15678/ [10.01.2021] 
EICHINGER, Ludwig M. (2008): Anglizismen im Deutschen meiden - warum das nicht so leicht ist. In: Moraldo, Sandro M. (Hrsg.): Sprachkontakt und Mehrsprachigkeit. Zur Anglizismendiskussion in Deutschland, Österreich, der Schweiz und Italien. Heidelberg, S. 69-93.

Eisenberg, Peter (2011): Das Fremdwort im Deutschen. Berlin; New York.

Glauninger, Manfred Michael / Graf, Martin Hannes (2009): Dialektale Aspekte der deutschen Fußballsprache in Österreich und der Schweiz. In: BurkhardT, Armin / Schlobinski, Peter (Hrsg.): Flickflack, Foul und Tsukahara: der Sport und seine Sprache. Mannheim; Leipzig; Wien; Zürich, S. 133-142.

MÜlLER, Stephan (2000): Deutsche Eishockey Meisterschaften. Libris Books on Demand.

PlancK, Karl (1898): Fußlümmelei. Über Stauchballspiel und englische Krankheit. Stuttgart. Zugänglich unter: https://www.deutschestextarchiv.de/book/view/planck_fussluemmelei_1898?p=7 [28.05.2021]

SCHNEIDER, Wolf (2008): Speak German! Warum Deutsch manchmal besser ist. Reinbek.

Sporkmann, Marcus / Zygowski, Peter (2005): Deutschlernen mit Kick. Berlin; München; Wien; Zürich; New York.

Stevenson, Angus (Hrsg.) (2010): Oxford Dictionary of English. Oxford.

Wesemeyer, Christin (2009): „Vorsicht, Kreis kommt!“ Zur Lexik der Handballsprache. In: BurKhaRdT, Armin / Schlobinski, Peter (Hrsg.): Flickflack, Foul und Tsukahara: der Sport und seine Sprache. Mannheim; Leipzig; Wien; Zürich, S. 211-228.

WinKLER, Marco (2018): Präzise, hart und eiskalt - die Lexik der Eishockeysprache. In: VaŇKOVÁ, Lenka (Hrsg): Fachlichkeit und Fachsprachlichkeit in varianten Kontexten. Berlin, S. 111-160.

Winkler, Marco (i. V.): Tore, Fallobst, tiefe Bänke - Metaphern \& Metonymien in der Sprache des Handballs, Eishockeys und Fußballs.

\section{Internetquellen:}

URL 1:https://www.eliteprospects.com/league/del [28.05.2021]

URL 2: https://www.eliteprospects.com/league/czech [28.05.2021]

URL 3: https://www.handball-world.news/o.red.r/news-1-1-1-51570.html [28.05.2021]

URL 4: https://www.thw-handball.de/de/news/weitere/2020/maerz/niklas-landin-daenisch-im-herzen-deutsch-im-kopf/ [28.05.2021] 